\title{
ASSOCIAÇÃO ENTRE ATIVIDADES OCUPACIONAIS DOS HANSENIANOS E INCAPACIDADES FÍSICAS*
}

Maria Helena Pessini de Oliveira**

\begin{abstract}
RESUMO: O referido trabalho pretendeu levantar as incapacidades físicas numa amostra de 218 portadores de hanseniase e associá-las às ocupações. Os resultados revelaram um elevado percentual de hansenianos exercendo trabalho que necessitam de esforços físicos para seu desempenho, expondo-se freqüentemente a traumatismos, acelerando assim o processo de instalação das incapacidades físicas. Observou-se também que $76,12 \%$ da amostra apresentam incapacidades físicas isoladas e/ou associadas, nas mãos, pés e olhos. Esta pesquisa foi realizada no período de 1990 a 1991.
\end{abstract}

ABSTRACT: The author intended to collect physical disabilities in a 218 leper population and to relate them to the occupations. The results revealed a high percentage of lepers whose jobs required physical effort, being often exposed to injuries, thus accelerating the process of physical disabilities. It was also observed that $76,12 \%$ of the sample presented isolated and/or related physical disabilities, in the hands, feet and eyes. This research took place between 1990 and 1991.

\section{INTRODUÇÃO}

A Hanseníase representa um sério problema de saúde pública, principalmente nas áreas endêmicas do mundo. As informações disponíveis sobre a prevalência de doença, na maioria dos países, não refletem a situação real, considerando que nem a procura dos dados, nem os relatos de casos atingem um nível desejável de informações. É uma doença que atinge com maior freqüência as classes sociais menor favorecidas, que residem em regiões pobres e isoladas $(4,8)$.

O meio sócio-econômico tem influenciado na distribuição das doenças de modo geral, pois a promiscuidade, favorecedora da multi-exposição, está ligada à pobreza e muitas vezes é determinante. A Hanseníase, como se sabe, vem ocorrendo com mais frequência nas classes menos favorecidas, cujas ocupações mal remuneradas e baixo padrão de vida econômico, contribuem para o desenvolvimento da doença. A falta de higiene, a subnutrição, a ignorância, além do inóculo infectante eficiente, a exposição suficiente e a receptividade do novo hospedeiro, são fatores constantes no "habitat" dos hansenianos e de seus comunicantes $(3,5)$.

Lamentavelmente, o mal de Hansen ainda é visto como uma doença estigmatizante, preconceituosa, refletindo para a sociedade a imagem de pessoas deformantes, com lesões cutâneas. Estas superstições em torno da hanseníase, trazem prejuízos consideráveis para o doente, que sof re pressões sociais através da rejeição, muitas vezes, pre judicado pela repulsa de que é vítima, e pelas manifestações que apresenta ${ }^{(6)}$.

A vivência com os hansenianos, tem mostrado que muitos portadores da enfermidade perdem seus empregos e são afastados de suas ocupações, antes mesmo de mostrarem diminuição da capacidade de produção. Tem-se observado que os doentes sofrem pressões sociais através da rejeição, dificultando o convívio social, até mesmo dentro da família ${ }^{(7)}$.

A repugnância da sociedade, em relação ao hanseniano, está associada às ameaças incapacitantes advindas da própria moléstia no decorrer da sua evolução.

\footnotetext{
* Trabalho apresentado como tema livre no $44^{\circ}$ Congresso Brasileiro de Enfermagem. Brasilia. D.F., 4 a 9 de outubro de 1992.

** Professora Doutora do Departamento Materno-Infantil e Saúde Pública da Escola de Enfermagem de Ribeirão Preto da Universidade de São Paulo.
} 
Alerta-se para a severidade, em relação a outras da doença, não podendo ser avaliada somente pelos números de pacientes existentes ou taxas de prevalência, mas sim pelos prejuízos decorrentes das incapacidades físicas a que submete seus portadores, bem como as consequências humanas e sociais para estes e suas famílias.

As incapacidades físicas constituem um problema no país, pelos índices elevados nas diversas regiões, e pela limitação excessiva da capacidade física, além do aspecto humano, relativo à dificuldade de atuação do doente, na sua vida profissional. Representa também perda significativa na força de trabalho dos países onde a doença é endêmica, além de causar repugnância na sociedade.

Segundo GARBINO(2), $70 \%$ a $85 \%$ dos pacientes portadores de hanseníase conhecidos, já apresentam alguma incapacidade física, e dentre elas, $30 \%$ são classificadas como graves.

Mediante o exposto neste estudo, propomos levantar as incapacidades físicas dos hansenianos $\mathrm{e}$ associá-las às atividades ocupacionais.

\section{MATERIAL E MÉTODO}

\subsection{População Amostral}

O total de portadores de hanseníase (859) que compareceram regularmente ao Centro de Saúde I, Centro de Saúde Escola e Centro de Saúde de Vila Virgínia,de 1990 a 1991, locais que oficialmente prestam assistência aos hansenianos na cidade de Ribeirão Preto, foram primeiramente listados de acordo com as formas clínicas Indeterminada (I), Tuberculóide (T), Virchowiana e Dimorfa (V ou D).

$\mathrm{O}$ total de indivíduos incluídos na amostra, nos três estratos (I, T, V ou D), foi determinado, a partir dos resultados teóricos, descritos em MENDEMHALL, OTT and SCHEAFFER (5), ficando estabelecido 218 pacientes assim distribuídos:

20 pacientes da forma clínica I,

36 pacientes da forma clínica $T$,

162 pacientes da forma clínica $\mathrm{V}$ ou $\mathrm{D}$.

Estabelecido o tamanho amostral em cada estrato, os pacientes listados forma sorteados aleatoriamente pelo processo casual simples. Tratou-se portanto de uma amostra estratificada, casual simples com distribuição ótima do tamanho.

Os hansenianos incluídos na amostra, após receberem consulta médica foram submetidos ao exame físico para levantar as incapacidades físicas nos olhos, mãos e pés, bem como outros dados pessoais relevantes no estudo. Adotou-se para o exame físico, o modelo de classificação das incapacidades físicas padronizadas pela OMS (Anexo).

\subsection{Definição e Classificação}

Para fins operaçionais, as ocupações desta investigação foram classificadas em categorias de $\mathrm{A}$ a E, tendo em vista, sobretudo, a intensidade do desprendimento da força para realização de determinada tarefa, bem como a habilidade e destreza requeridas para o seu desempenho.

Entende-se que qualquer atividade para se realizar, impõe uma conjugação do intelecto e do físico, dependente pois, da articulação de mãos, braços, pés, pernas, olhos e da mente para a consecução de seu intento.

Considerando-se a população investigada e o objetivo de verificar as relações existentes entre os tipos de ocupações do hanseniano e os graus das incapacidades físicas apresentados, foi construída uma tipologia das ocupacões encontradas, cujo critério fundamental, como se disse, liga-se às atividades que se caracterizam pela necessidade do uso de força física ou de habilidade manual.

\section{TIPOLOGIA DAS OCUPAÇÕES ENCONTRADAS}

Categoria A: inclui-se toda e qualquer atividade que necessite de um grande desprendimento de força física "trabalho pesado": lavrador, carregador de sacos, entregados de gás, servente de pedreiro, lenhador.

Categoria B: foram aí incluídas todas as atividades que necessitam de moderado desprendimento de força física, aliado à exigência de algum conhecimento semi-especializado: pedreiro, mecânico, encanador, marceneiro, carpinteiro, eletricista, marmorista, vidraceiro, ladrilheiro, serralheiro, fiandeiro de algodão, fiambreiro, macarroneiro, motorista de caminhão, moxambeiro, empregada doméstica e dona de casa, faxineira, lavadeira.

Categoria C: foram incluídas neste ítem todas as atividades que exigem habilidade manual vinculada a um corpo de conhecimento técnico: modelista de calçado, modelista de roupa, costureira, bordadeira, conf eiteira, manicure, barbeiro, auxiliar de escritório.

Categoria D: foram aí incluídas as atividades 
que não necessitam de habilidades manuais específicas caracterizando-se, ainda, por um pequeno desprendimento de força física: fiscal agrícola, corretor de imóveis, telefonista, balconista, vendedor de roupa, assistente religiosa, vigilante noturno, porteiro, vendedor ambulante.

APOSENTADO: toda e qualquer pessoa que recebe remuneração por direitos sobre o tempo limitado de seu trabalho ou por invalidez, isto é, por se encontrar sem condições físicas, para executar sua ocupação.

\section{CLASSIFICAÇÃO DAS INCAPACIDADES FÍSICAS}

- Incapacidade física isolada: considerou-se incapacidade física isolada, lesões incapacitantes que ocorreram nuna única localização, ou seja, só nas mãos, só nos pés ou só nos olhos.

- Incapacidade física associada: considerou-se incapacidade física associada, lesões incapacitantes que ocorreram em mais de uma localização, ou seja, mãos e pés, mãos e olhos, pés e olhos, ou mãos, pés e olhos.

- Tipo de incapacidade física: considerou-se tipos de incapacidades físicas o conjunto de lesões incapacitantes que se desenvolveram nas mãos, pés e olhos. Diante de sua variação e gravidade, foram classificadas em graus I, II e III.

- Incapacidade física de grau I: foram assim classificados todo o paciente que apresentou, durante o exame físico, sinais de insensibilidade nas mãos, pés direito e esquerdo, e conjuntivite nos olhos direito e esquerdo.

- Incapacidade física de grau II: foram classificados todo o paciente que apresentou um ou mais de comprometimento neurológico assim especificado:

1) mãos direita e esquerda: úlcera e traumas, garra móvel e reabsorção leve;

2) pés direito e esquerdo: úlceras tróficas, garra de artelhos, pé caído e reabsorção leve;

3) olhos direito e esquerdo: lagoftalmo, irites ou ceratite e visão embaraçada.

- Incapacidade física de grau III: foram classificados todo o paciente que apresentou um ou mais sinal de comprometimento neu- rológico assim especificado:

1) mãos direita e esquerda: mão caída, articulação endurecida e reabsorção grave;

2) pés direito e esquerdo: contratura e reabsorção grave;

3) olhos direito e esquerdo: grave perda da visão e cegueira.

\section{ANÁLISE DOS RESULTADOS}

Trabalhou-se com uma amostra de pacientes constituída de $57,34 \%$ de homens e $42.63 \%$ de mulheres, com maiores percentuais entre os grupos etários de 35 a 65 anos $(66,95 \%)$.

A média de idade entre os homens foi 49,8 anos, enquanto as mulheres corresponderam à média de 51,7 anos de idade. Nota-se portanto, que a doença congrega um grande número de pessoas com idade elevada, demonstrando a cronicidade da mesma, exigindo um tratamento mais demorado nas formas clínicas multibacilares ${ }^{(1)}$.

Os resultados relativos às ocupações (Tabela 1 ), mostram um elevado percentual de pacientes exercendo trabalho que necessita de esforços físicos para seu desempenho, expondo-se freqüentemente a traumatismos, acelerando assim o processo de instalação das incapacidades físicas. Observa-se a diminuição acentuada das ocupações que os hansenianos exerciam antes do aparecimento da doença (categorias A e B) frente às atividades referidas no momento do exame físico, e a maior proporção de homens aposentados por invalidez com relação às mulheres.

Trata-se portanto, de uma população com maior contingente de assalariados manuais (categorias B e A), portadores ou não de conhecimento especializado, onde as mulheres pertenciam a categoria B, padrão doméstico, seja por atividades cońcernentes à vida familiar (do lar), ou na condição de servir mediante um pagamento (empregada doméstica), sem direito a aposentadoria. Chama a atenção o número de pacientes executando atividades da categoria $\mathrm{D}$, para ambos os sexos. As mudanças de ocupações podem ser justificadas pelo estígma e preconceito que ainda envolvem a hanseníase, seja por percepção do próprio paciente, ou por exigências do meio.

A categoria de aposentado, à qual pertence uma parte da amostra do presente trabalho (36,23\%), leva à crença de uma auto-suficiência econômica. Conhecendo, porém, o nível dessa remuneração em nosso 
Tabela 1 - Distribuição dos pacientes com hanseníase, segundo o sexo e as ocupações que exerciam antes do diagnóstico clínico, no momento da realização da pesquisa.

\begin{tabular}{|c|c|c|c|c|c|c|c|c|}
\hline \multirow{3}{*}{$\begin{array}{l}\text { Categoria } \\
\text { Ocupacional }\end{array}$} & \multicolumn{4}{|c|}{$\frac{\text { Antes do Diagnóstico Clínico }}{\text { Sexo }}$} & \multicolumn{4}{|c|}{$\frac{\text { No Momento da Pesquisa }}{\text { Sexo }}$} \\
\hline & \multicolumn{2}{|c|}{ Masculino } & \multicolumn{2}{|c|}{ Feminino } & \multicolumn{2}{|c|}{ Masculino } & \multicolumn{2}{|c|}{ Feminino } \\
\hline & $\mathrm{N}^{\circ}$ & $\%$ & $\mathrm{~N}^{\circ}$ & $\%$ & $\mathrm{~N}^{\circ}$ & $\%$ & $\mathrm{~N}^{0}$ & $\%$ \\
\hline A & 44 & 35,20 & 13 & 13,97 & 8 & 6,40 & 5 & 5,37 \\
\hline B & 73 & 58,40 & 75 & 80,64 & 27 & 21,60 & 76 & 81,72 \\
\hline C & 2 & 1,60 & 4 & 4,30 & 5 & 4,00 & - & - \\
\hline D & 6 & 4,80 & 1 & 1,07 & 15 & 12,00 & 3 & 3,22 \\
\hline$E$ & - & & - & & 70 & 56,00 & 9 & 9,67 \\
\hline TOTAL & 125 & 100,00 & 93 & 99,98 & 125 & 100,00 & 93 & 99,98 \\
\hline
\end{tabular}

Categoria A: Grande desprendimento de força "trabalho pesado".

Categoria B: Moderado desprendimento de força e conhecimento semi-especializado.

Categoria C: Habilidade manual vinculada ao conhecimento técnico.

Categoria D: Não necessita de habilidade manual específica, pequeno desprendimento de força, maior ou menor nivel intelectual.

Categoria E: Afastado de suas funções por invalidez - aposentado.

país, insuficiente para um padrão de vida desejável a qualquer ser humano, pode-se refletir sobre suas consequências, seja do ponto de vista de sua sobrevivência, seja para atenuar seus conflitos em relação à saúde e adaptação à comunidade.

$\mathrm{O}$ alto índice das incapacidades físicas encontradas na amostra (Tabela 2), retrata uma população marcada pela doença, especialmente pelo descuido a que está sujeita. Os dados revelam que grande parte dos pacientes apresentam uma ou mais incapacidades físicas isoladas e associadas entre mãos, pés e olhos, com maior frequência entre a idade de 35-65 anos, significando uma perda bio-psico-social considerável para o doente, família e sociedade e abrangendo o período de maior produtividade de trabalho do indivíduo.
As lesões incapacitantes podem estar relacionadas às consequências de um diagnóstico tardio, seja pela ineficiência dos serviços de saúde, à qualidade da vigilância dos comunicantes, ou pelo descuido e falta de conhecimento sobre a moléstia ${ }^{(8)}$. Agravando a situação do hanseniano, há o preconceito que ainda prevalece não só na sociedade, mas entre os profissionais de saúde.

Na Tabela 3, observa-se que as incapacidades físicas das mãos, pés e olhos, ocorreram em ambos os sexos. Entretanto, quando comparada às incapacidades físicas associadas, observa-se uma diferença de $8,92 \%$ a mais para o sexo masculino, confirmando achados anteriores.

Analisando as ocorrências das incapacidades fí-

Tabela 2 - Distribuição dos pacientes com Hanseníase, segundo a faixa etária e a presença ou não de incapacidades físicas isoladas ou associadas, nas mãos e/ou nos pés e/ou nos olhos.

\begin{tabular}{|c|c|c|c|c|c|c|c|c|c|c|c|c|}
\hline \multirow{4}{*}{$\begin{array}{l}\text { Grupo } \\
\text { Etário }\end{array}$} & \multicolumn{12}{|c|}{ Incapacidades Físicas } \\
\hline & \multicolumn{8}{|c|}{ Isoladas } & \multirow{2}{*}{\multicolumn{2}{|c|}{$\begin{array}{c}\text { Associadas } \\
\text { Graus I e/ou II } \\
\text { e/ou III }\end{array}$}} & \multirow{2}{*}{\multicolumn{2}{|c|}{ Total }} \\
\hline & \multicolumn{2}{|c|}{ Ausência } & \multicolumn{2}{|c|}{ Grau I } & \multicolumn{2}{|c|}{ Grau II } & \multicolumn{2}{|c|}{ Grau III } & & & & \\
\hline & $\mathrm{N}^{0}$ & $\%$ & $\mathrm{~N}^{0}$ & $\%$ & $\mathrm{~N}^{\circ}$ & $\%$ & $\mathrm{~N}^{\circ}$ & $\%$ & $\mathrm{~N}^{\circ}$ & $\%$ & $\mathrm{~N}^{0}$ & $\%$ \\
\hline $15 \longmapsto-25$ & 8 & 3,66 & 1 & 0,45 & - & - & - & - & 5 & 2,29 & 14 & 6,42 \\
\hline $25 \longmapsto 35$ & 8 & 3,66 & 3 & 1,37 & - & - & - & - & 8 & 3,66 & 19 & 8,71 \\
\hline $35 \longmapsto-45$ & 17 & 7,79 & 4 & 1,83 & 4 & 1,83 & - & - & 16 & 7,33 & 41 & 18,80 \\
\hline $45 \longmapsto 55$ & 11 & 5,04 & 5 & 2,29 & 5 & 2,29 & 2 & 0,91 & 30 & 13,76 & 53 & 24,31 \\
\hline $55 \longmapsto-65$ & 4 & 1,83 & 1 & 0,45 & 2 & 0,91 & 3 & 1,37 & 42 & 19,26 & 52 & 23,85 \\
\hline $65 \longmapsto 75$ & 4 & 1,83 & - & - & 4 & 1,83 & 4 & 1,83 & 13 & 5,96 & 25 & 11,46 \\
\hline $75 \longmapsto 85$ & - & - & - & - & 1 & 0,45 & 1 & 0,45 & 11 & 5,04 & 13 & 5,96 \\
\hline $85 \longmapsto$ & - & - & - & - & - & & - & & 1 & 0,45 & 1 & 0,45 \\
\hline TOTAL & 52 & 23,85 & 14 & 6,42 & 16 & 7,33 & 10 & 4,58 & 126 & 57,79 & 218 & 99,97 \\
\hline
\end{tabular}


Tabela 3 - Distribuição dos pacientes com Hanseníase, segundo o sexo e a presença ou não de incapacidades físicas isoladas ou associadas, nas mãos e/ou nos pés e/ou nos olhos.

\begin{tabular}{|c|c|c|c|c|c|c|c|c|c|c|c|c|}
\hline \multirow{4}{*}{ Sexo } & \multicolumn{12}{|c|}{ Incapacidades Físicas } \\
\hline & \multicolumn{8}{|c|}{ Isoladas } & \multirow{2}{*}{\multicolumn{2}{|c|}{$\begin{array}{c}\text { Associadas } \\
\text { Graus I e/ou II } \\
\text { e/ou III }\end{array}$}} & \multirow{2}{*}{\multicolumn{2}{|c|}{ Total }} \\
\hline & \multicolumn{2}{|c|}{ Ausência } & \multicolumn{2}{|c|}{ Grau I } & \multicolumn{2}{|c|}{ Grau II } & \multicolumn{2}{|c|}{ Grau III } & & & & \\
\hline & $\mathrm{N}^{0}$ & $\%$ & $\mathrm{~N}^{0}$ & $\%$ & $\mathrm{~N}^{\circ}$ & $\%$ & $\mathrm{~N}^{0}$ & $\%$ & $\mathrm{~N}^{0}$ & $\%$ & $\mathrm{~N}^{0}$ & $\%$ \\
\hline Fem. & 24 & 25,80 & 8 & 8,60 & 7 & 7,52 & 5 & 5,37 & 49 & 52,68 & 93 & 99,97 \\
\hline Masc. & 28 & 22,40 & 6 & 4,80 & 9 & 7.20 & 5 & 4.00 & 77 & 61,60 & 125 & 100.00 \\
\hline TOTAL & 52 & 23,85 & 14 & 6,42 & 16 & 7,33 & 10 & 4,58 & 126 & 57,79 & 218 & 99,97 \\
\hline
\end{tabular}

sicas, dentro de cada categoria profissional, observase que a categoria $\mathrm{E}$ (aposentado), concentra os maiores valores percentuais de pacientes portadores de uma ou mais lesões incapacitantes (Tabela 4).

Os referidos resultados permitem levantar a hipótese de que as ocupações executadas pelos hansenianos, principalmente as da categoria $\mathrm{B}$ e A, podem ter contribuído com o agravamento do quadro incapacitante, por serem, segundo dados obtidos, as maiores responsáveis pelo número de pacientes aposentados. (Tabela 1). Ademais, as alterações físicas, decorrentes da própria doença, podem conduzir o desempenho inadequado de atividades que exigem maior participação das mãos, pés e olhos.

Possivelmente, seja esta uma das razões que levam os pacientes a optarem por outras ocupações, conforme as definidas na categoria $\mathrm{D}$.

No aspecto profissional, as possibilidades de trabalho e independência econômica estão limitadas, não somente pela enfermidade em si, mas também pela incapacidade do ponto de vista funcional determinada pelas suas características deformantes. As ocupações profissionais dos hansenianos podem gerar lesões incapacitantes, enquanto as alterações neurológicas, provenientes da doença, podem dificultar o desempenho de determinadas atividades. Os pacientes acabam desenvolvendo movimentos laboriais apropriados a situação, acentuando ainda mais as lesões deformantes ${ }^{(6)}$.

Ademais, deve-se considerar que o estigma da deformidade, leva à discriminação no ambiente de trabalho, na família e no grupo social; outrossim, a perda parcial da capacidade de trabalho do hanseniano, conduz à desintegração do processo bio-psico-social do mesmo.

Questiona-se o quanto estes pacientes aposentados tornam-se onerosos para os gastos públicos, além dos próprios prejuízos físicos e psico-sociais de que são vítimas. Esta situação poderia, na maioria das vezes, ser evitada, se os doentes contassem com serviços estruturados, capazes de desenvolver assistência integral, dispondo de pessoal qualificado para um trabalho educativo, conscientizando a população doente quanto importância de prevenção das incapacidades, mediante técnicas simples, acessíveis a qualquer instituição de saúde e ao próprio indivíduo.

A prevenção e tratamento das incapacidades físicas no doente, se converte na peça mais importante para o combate e controle da hanseníase, por ser esta a raiz do estigma na sociedade. Através de sua prevenção pode-se reincorporar o indivíduo à sociedade como ser útil, produtivo, mantendo-o no campo de trabalho.

O afastamento do doente de suas ocupações cria

Tabela 4 - Distribuição dos pacientes com Hanseníase, segundo as ocupações e a presença ou não de incapacidades físicas isoladas ou associadas, nas mãos e/ou nos pés e/ou nos olhos

\begin{tabular}{|c|c|c|c|c|c|c|c|c|c|c|c|c|}
\hline \multirow{4}{*}{$\begin{array}{l}\text { Categoria } \\
\text { Ocupacional }\end{array}$} & \multicolumn{12}{|c|}{ Incapacidades Físicas } \\
\hline & \multicolumn{8}{|c|}{ Isoladas } & \multirow{2}{*}{\multicolumn{2}{|c|}{$\begin{array}{c}\text { Associadas } \\
\text { Graus I e/ou II } \\
\text { e/ou III }\end{array}$}} & \multirow{2}{*}{\multicolumn{2}{|c|}{ Total }} \\
\hline & \multicolumn{2}{|c|}{ Ausência } & \multicolumn{2}{|c|}{ Grau I } & \multicolumn{2}{|c|}{ Grau II } & \multicolumn{2}{|c|}{ Grau III } & & & & \\
\hline & $\mathrm{N}^{\circ}$ & $\%$ & $\mathrm{~N}^{\circ}$ & $\%$ & $\mathrm{~N}^{\circ}$ & $\%$ & $\mathrm{~N}^{\circ}$ & $\%$ & $\mathrm{~N}^{\circ}$ & $\%$ & $\mathrm{~N}^{\circ}$ & $\%$ \\
\hline A & 4 & 30,76 & - & & 1 & 7,69 & - & - & 8 & 61,53 & 13 & 99,98 \\
\hline B & 32 & 31,06 & 10 & 9,70 & 8 & 7,76 & 5 & 4,85 & 48 & 46,60 & 103 & 99,97 \\
\hline C & 3 & 60,00 & - & & - & & - & & 2 & 40,00 & 5 & 100,00 \\
\hline D & 3 & 16,66 & 3 & 16,66 & 1 & 5,55 & 1 & 5,55 & 10 & 55,55 & 18 & 99,97 \\
\hline$E$ & 10 & 12,65 & 1 & 1,26 & 6 & 7.59 & 4 & 5.06 & 58 & 73,41 & 79 & 99.97 \\
\hline TOTAL & 52 & 23,85 & 14 & 6.42 & 16 & 7.33 & 10 & 4.58 & 126 & 57.79 & 218 & 99,97 \\
\hline
\end{tabular}


problemas que acabam interferindo no relacionamento e na harmonia familiar, contribuindo direta ou indiretamente para os desajustes psicológicos. Somase ainda, a análise dos critérios empregados para o afastamento dos pacientes de suas ocupações, em especial, o rótulo "aposentado por invalidez", termo bastante discutível. A vivência com os hansenianos tem revelado que pacientes mutilados conseguem exercer funções especializadas como encanador, eletricista, etc, enquanto outros reabilitados, encontram dificuldades para se adaptar ao emprego.

O processo de prevenção de incapacidade, a reintegração do hanseniano na sociedade, depende muito do próprio "querer" do paciente, da credibilidade e aceitação da proposta de trabalho que o profissional venha desenvolver com ele, e até da sua própria aceitação como portador de hanseníase.

Agravando a situação do hanseniano, há o preconceito que ainda prevalece na sociedade, entre os próprios doentes e nos profissionais de saúdc.

$\mathrm{O}$ enf ermeiro, como membro participante e ativo da equipe de saúde, tem competência profissional, para desenvolver junto aos serviços de saúde, os programas destinados aos hansenianos. $\mathrm{O}$ atendimento de enfermagem aproxima o profissional, pernitindo oportunidades de compartilhar o problema, refletir sobre as peculiaridades que envolvem o hanseniano no seu dia a dia, e com isso as orientações se centralizarem nos fatos levantados. Orientar o paciente sobre os problemas decorrentes de sua doença, sem a priori, considerar a multicausalidade, é repetir informações teóricas, sem o envolvimento com a questão em si, e isso pode levar a resultados frustrantes.

Trabalhar com hanseniano não é restringir suas atividades impondo conhecimentos e exigindo mu- danças de comportamento "adequados" à ótica do enfermeiro. É compartilhar, evitando unilateralidades.

Urge refletir sobre a situação atual do controle da doença. a filosofia assistencial vigente direcionada para o paciente, família e comunidade, bem como a abordagem do assunto, a importância da inclusão do hanseniano na elaboração dos trabalhos da instituição de saúde, quanto à prevenção, sua reabilitação, tratamento, seguimento e o envolvimento da equipe profissional. Esta é uma questão coletiva.

\section{CONCLUSÃO}

Os resultados relativos às ocupações mostraram um elevado percentual de hansenianos exercendo ocupações que necessitam de esforços físicos para seu desempenho, expondo-se freqüentemente a traumatismos, acelerando o processo de instalação das incapacidades físicas. É significativo o número de pacientes que deixaram de realizar as ocupações anteriores ao aparecimento da doença, substituindo por outras atividades inferiores e mal remuneradas.

As incapacidades fisicas constituem um problema complexo, af etando não só o físico, mas o psico e o social, dificultando a reabilitação do hanseniano. Os obstáculos crescem à medida que se acentuam as alterações físicas, e quanto mais o paciente conhecer sobre sua doença, mais facilidade encontrará para compreender a sua participação no processo de prevenção e reabilitação.

Por outro lado, a Hanseníase deve ser encarada de forma ampla, abrangendo o doente como um todo, uma vez que a doença representa um complexo biopsico-social, responsável muitas vezes, pelo afastamento de suas atividades ocupacionais.

\section{REFERÊNCIAS BIBLIOGRÁFICAS}

1. CRISTOFOLINI, L. Pacientes em quinioterapia anti-hansenica: problemas nasais. Tese de Doutoramento apresentada na Escola de Enfermagem da USP. São Paulo, 1991.

2. GARBINO, J.A. Gènese das incapacidades em Hanseniase. Bauru: Inst. Lauro de Souza Lima. Centros de Estudos Dr. Reynaldo Quaglio. 1991.

3. JORNAL DO MORHAN. Movimento de Reintegração do Hanseniano. Ano IX, n.17, São Bernardo do Campo, junho, 1990

4. LOMBARDI, C., FERREIRA, J., MOTTA, C., OLIVEIRAL, M.L. Hanseniase - Epidemiologia e Controle. São Paulo: Convênio IMESP/SAESP. 1990.
5. MENDENHALL, W., OTT, L., SHEAFFER, R.L. Elementary survey sampling. Duxbury Press, a Division of W adsworth Publishing - Co., Inc. Belmont, c.a. 1971.

6. OLIVEIRA, M.H.P. Incapacidades Fisicas em Portadores de Hanseniase. Tese de Doutoramento apresentada na Escola de Enf ermagem de Ribeirão Preto - USP. Ribeirão Preto, SP, 1987.

7. OLIVEIRA, M.H.P. A percepção dos hansenianos quanto à sua doença. In: SIBRASEn, $3^{\circ}$, Ribeirão Preto, 13 a 15 maio, 1992. Anais... Ribeirão Preto, EERP/USP, 1992. p.444-455.

8. ROMERO, A. Políticas y estratégias para los programas de control de la lepra. Boletin de la Oficina Sanitária Panamericana, v.93, p.220-231, 1982.

Recebido para publicação em 30.08 .93 


\section{ANEXO}

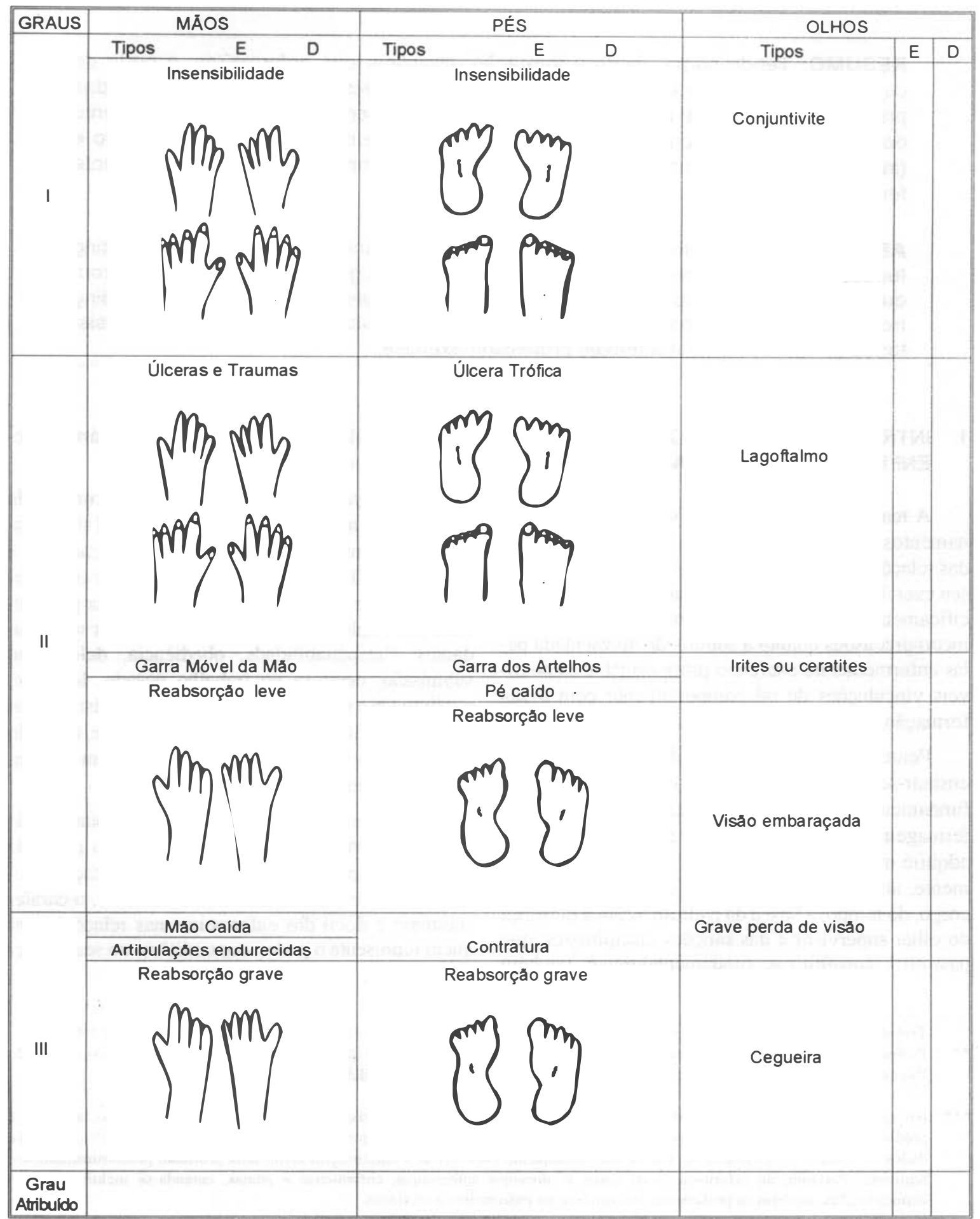

